\title{
PU.1 controls the expression of long noncoding RNA HOTAIRM1 during granulocytic differentiation
}

\author{
Shuyong Wei ${ }^{1}$, Ming Zhao ${ }^{1}$, Xiaoling Wang ${ }^{1}$, Yizhen $\mathrm{Li}^{1}$ and Kankan Wang ${ }^{1,2^{*}}$
}

\begin{abstract}
Background: Long noncoding RNA HOX antisense intergenic RNA myeloid 1 (HOTAIRM1) has been characterized as a critical factor in all-trans retinoic acid (ATRA)-induced differentiation of acute promyelocytic leukemia (APL) cells. However, the essential transcription factor for gene expression of HOTAIRM1 is still unknown.

Findings: Chromatin immunoprecipitation (ChIP) assays revealed that PU.1 constitutively bound to the regulatory region of HOTAIRM1. Co-expression of PU.1 led to the transactivation of the regulatory region of HOTAIRM1 in a reporter assay. Detailed analysis showed that two PU.1 motifs, which were located around +1100 bp downstream of the transcriptional start site of the HOTAIRM1 promoter, were responsible for the PU.1-dependent transactivation. The induction of HOTAIRM1 by ATRA was dependent on PU.1, and ectopic expression of PU.1 significantly upregulated HOTAIRM1. Furthermore, low HOTAIRM1 expression was observed in APL cells, which was attributed to the reduced PU.1 expression rather than the repression by PML-RARa via the direct binding.

Conclusion: PU.1 directly activates the expression of HOTAIRM1 through binding to the regulatory region of HOTAIRM1 during granulocytic differentiation. The reduced PU.1 expression, rather than PML-RARa itself, results in the low expression of HOTAIRM1 in APL cells. Our findings enrich the knowledge on the regulation of IncRNAs and the underlying mechanisms of the abnormal expression of IncRNAs involved in APL.
\end{abstract}

Keywords: HOTAIRM1, PU.1, APL, PML-RARa, Transcriptional regulation

\section{Introduction}

Recently, it has become increasingly clear that long noncoding RNAs (lncRNAs) function as a class of versatile regulators through interaction with DNA, RNA, and proteins to modulate gene expression [1-3]. Extensive studies have focused on the functions of IncRNAs in cells and demonstrated that IncRNAs modulate transcriptional regulation, regulate protein activities, play structural or organizational roles, and serve as precursors to small RNAs [4-7]. Thus, aberrant expression of IncRNAs is associated with disease development. However, the molecular mechanisms underlying the regulation of lncRNAs are still largely unknown.

\footnotetext{
* Correspondence: kankanwang@shsmu.edu.cn

${ }^{1}$ State Key Laboratory of Medical Genomics and Shanghai Institute of Hematology, Ruijin Hospital, Shanghai Jiao Tong University School of Medicine, 197 Ruijin Er Rd, Shanghai 200025, China

${ }^{2}$ Sino-French Research Center for Life Sciences and Genomics, Ruijin Hospital, Shanghai Jiao Tong University School of Medicine, Shanghai 200025, China
}

HOX antisense intergenic RNA myeloid 1 (HOTAIRM1) is a myeloid-specific IncRNA, which is transcribed between the HOXA1 and HOXA2 genes. Functional studies have revealed that HOTAIRM1 is essential for myeloid differentiation. HOTAIRM1 knockdown impairs all-trans retinoic acid (ATRA)-driven granulocytic differentiation and attenuates the expression of differentiation-related genes including ITGAM, CD18, HOXA1, and HOXA4 [8]. HOTAIRM1 knockdown can also result in a reduction of ATRA-induced cell cycle arrest at the transition from G1 to $S$ phase [9]. A recent clinical study has investigated the clinical impact of HOTAIRM1 and demonstrated that high HOTAIRM1 expression is associated with poor prognosis in intermediate-risk acute myeloid leukemia (AML) patients [10]. In addition, the low expression of HOTAIRM1 is observed in acute promyelocytic leukemia (APL) [10]. Despite the importance of HOTAIRM1 in myeloid differentiation, the critical transcription factor regulating the expression of HOTAIRM1 has not been identified to date. 
Transcription factor PU.1 is a master regulator in the control of myeloid differentiation [11-13]. PU.1 transcriptionally regulates the genes necessary for myeloid differentiation of granulocytes and monocytes, including granulocyte/macrophage colony-stimulating factor receptor (GM-CSFR) [14-16], CD11b [17], CD18 [18], and myeloperoxidase [19]. A tightly regulated level of PU.1 is essential for normal hematopoiesis. Dysregulation of PU.1 can lead to the development of AML [20-22]. Our previous work $[23,24]$ showed that, in the development of APL, both the targets and the expression level of PU.1 are inhibited by the oncogenic PML-RAR $\alpha$ fusion protein, which is generated by the chromosomal translocation, $t(15 ; 17)$. Afterwards, a series of PU.1-regulated genes, such as genes encoding HCK [25] and immunoproteosomes [26], have been reported to contribute to APL leukemogenesis due to the dysregulation of their expression. During the treatment of APL, ATRA restores the expression of both PU.1 and its downstream target genes by degrading PML-RAR $\alpha$, thus relieving the differentiation block [27-29]. Moreover, genome-wide binding profiling of these APL-associated transcription factors, e.g., PML-RAR $\alpha$ and PU.1, has demonstrated that the molecular consequences of these transcription factors are undoubtedly complex, as they associate with and likely regulate a relatively large number of diverse genes accounting for as much as $10-15 \%$ of the genome [30]. Those target genes include not only protein-coding genes but also noncoding transcripts, especially lncRNAs.

In this study, using chromatin immunoprecipitation (ChIP) and luciferase reporter assays, we found that PU.1 bound to the regulatory region of HOTAIRM1 in vivo and transactivated this region, which was mainly mediated through two PU.1 motifs around $+1100 \mathrm{bp}$ downstream to the transcription start site (TSS). Furthermore, PU.1 knockdown down-regulated HOTAIRM1 and PU.1 overexpression increased HOTAIRM1. Finally, we revealed that the reduced PU.1 expression was responsible for the low HOTAIRM1 expression in APL cells.

\section{Results}

The induction of HOTAIRM1 upon ATRA treatment requires intermediate protein synthesis

It has been shown that HOTAIRM1 is up-regulated in APL cells upon exposure to ATRA and plays a critical role in myeloid differentiation [8]. Since HOTAIRM1 has two variants, we designed variant-specific primers to examine the expression of each variant in APL-derived NB4 cells upon ATRA treatment. As shown in Fig. 1a, HOTAIRM1 variant 2 was the major isoform that was expressed both before and after ATRA treatment. By comparison, HOTAIRM1 variant 1 was far less abundant than variant 2 . Both variants were up-regulated upon ATRA treatment. To investigate whether the induction of HOTAIRM1 was a direct effect of ATRA, NB4 cells were pretreated with a translation inhibitor cycloheximide (CHX) for $30 \mathrm{~min}$ prior to the addition of ATRA to interfere with the translation process of newly synthesized proteins. As shown in Fig. 1b, CHX significantly impaired the up-regulation of both HOTAIRM1 variants by ATRA, suggesting that the induction of HOTAIRM1 requires newly synthesized intermediate protein(s).

\section{PU.1 directly binds to the regulatory region of HOTAIRM1}

To search for the newly synthesized regulatory factors involved in the up-regulation of HOTAIRM1, we first identified the regulatory region responsible for the transcriptional regulation of HOTAIRM1, which can be defined using binding profiles of H3K4me3 and H3K27Ac. Due to the myeloid-specific expression of HOTAIRM1, we chose CD14+ monocytes (a myeloid lineage) for the analysis. We utilized the chromatin immunoprecipitation (ChIP)-seq data generated by the ENCODE Project Consortium [31]. The binding profiles were displayed using the UCSC Genome Browser (http://genome.ucsc.edu)
A

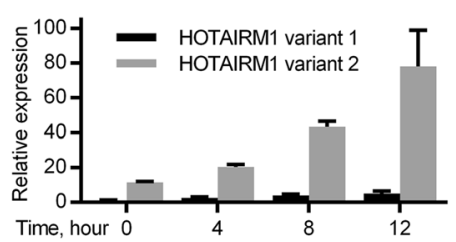

B

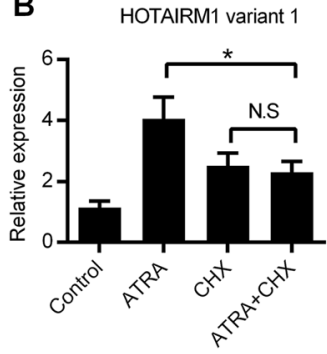

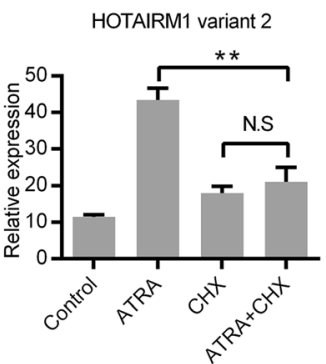

Fig. 1 The induction of HOTAIRM1 upon ATRA treatment requires the intermediate protein synthesis. a The expression levels of two HOTAIRM1 variants at different time points following $1 \mu \mathrm{M}$ ATRA treatment in NB4 cells. $\mathbf{b}$ The expression levels of two HOTAIRM1 variants in NB4 cells before and after ATRA treatment $(1 \mu \mathrm{M})$ for $8 \mathrm{~h}$, with or without 30 min $\mathrm{CHX}$ pretreatment $(100 \mu \mathrm{g} / \mathrm{mL})$. The relative expression level was calculated relative to that of variant 1 at $0 \mathrm{~h}$. An average of two control genes (GAPDH and ACTB) was used for normalization. The data represent the mean of three replicates $\pm S D .{ }^{*} p<0.05 ;{ }^{*} p<0.01$ 
[32]. As shown in Fig. 2a, the enrichment of H3K4me3 and H3K27Ac binding was found to be located from about -100 bp upstream of the transcription start site (TSS) to approximately $+1200 \mathrm{bp}$ downstream of the TSS. The data indicated that this region mediated the transcription of HOTAIRM1 and this region was used in the following experiment and analysis. We then investigated the significantly enriched transcription factor binding sites within this regulatory region of HOTAIRM1 using Matinspector [33]. Interestingly, we found eight putative binding sites for PU.1 (Fig. 2b), which is a hematopoietic transcription factor involved in myeloid differentiation and up-regulated upon ATRA treatment. To analyze the binding of PU.1, we performed ChIP assays in ATRA-treated NB4 cells. We designed six primer pairs to search for the in vivo binding of PU.1 around these eight PU.1-motif-containing sites. The closely located motifs were investigated using the same set of the primers. The primer pair " +600 " covered motifs at +586 and +629 and the primer pair " $+1100 "$ covered motifs at +1067 and +1123 . The regions upstream $(-400)$ and downstream $(+1600)$ of the regulatory region and an irrelevant region $(\mathrm{NC})$ were used as negative controls. As shown in Fig. 2c, the position around +1100 bp downstream to the TSS showed dramatically significant enrichment of PU.1 binding in NB4 cells, and this was far more noticeable than the other putative sites. Additionally, we also detected the PU.1 binding in another myeloid cell line, U937, which belongs to a different AML subtype but can also undergo myeloid differentiation upon ATRA treatment. The similar PU.1 binding pattern was observed in ATRA-treated U937 cells, ruling out cell line-specific phenomena. The data together indicated that PU.1 directly bound to the regulatory region of HOTAIRM1.

\section{PU.1 transactivates HOTAIRM1 through the +1100 region in the HOTAIRM1 promoter}

To further investigate whether PU.1 transactivates the regulatory region of HOTAIRM1 through the PU.1 binding sites identified above, we employed the luciferase reporter assay in HEK-293T cells, which lack endogenous PU.1. We cloned a $1370 \mathrm{bp}$ fragment of the regulatory region of HOTAIRM1 spanning from -134 to +1237 into a luciferase reporter plasmid, which was designated as pGL3-HOTAIRM1 (Fig. 3a). We also included one mutant form of the complete regulatory region in which the core sequences of PU. 1 sites at +1067 and +1123 were mutated from GGAA to CCAA, and one truncated construct spanning from -134 to +911 and lacking PU.1 sites on the +1100 region. As shown in Fig. 3b, coexpression of PU.1 resulted in significant activation of

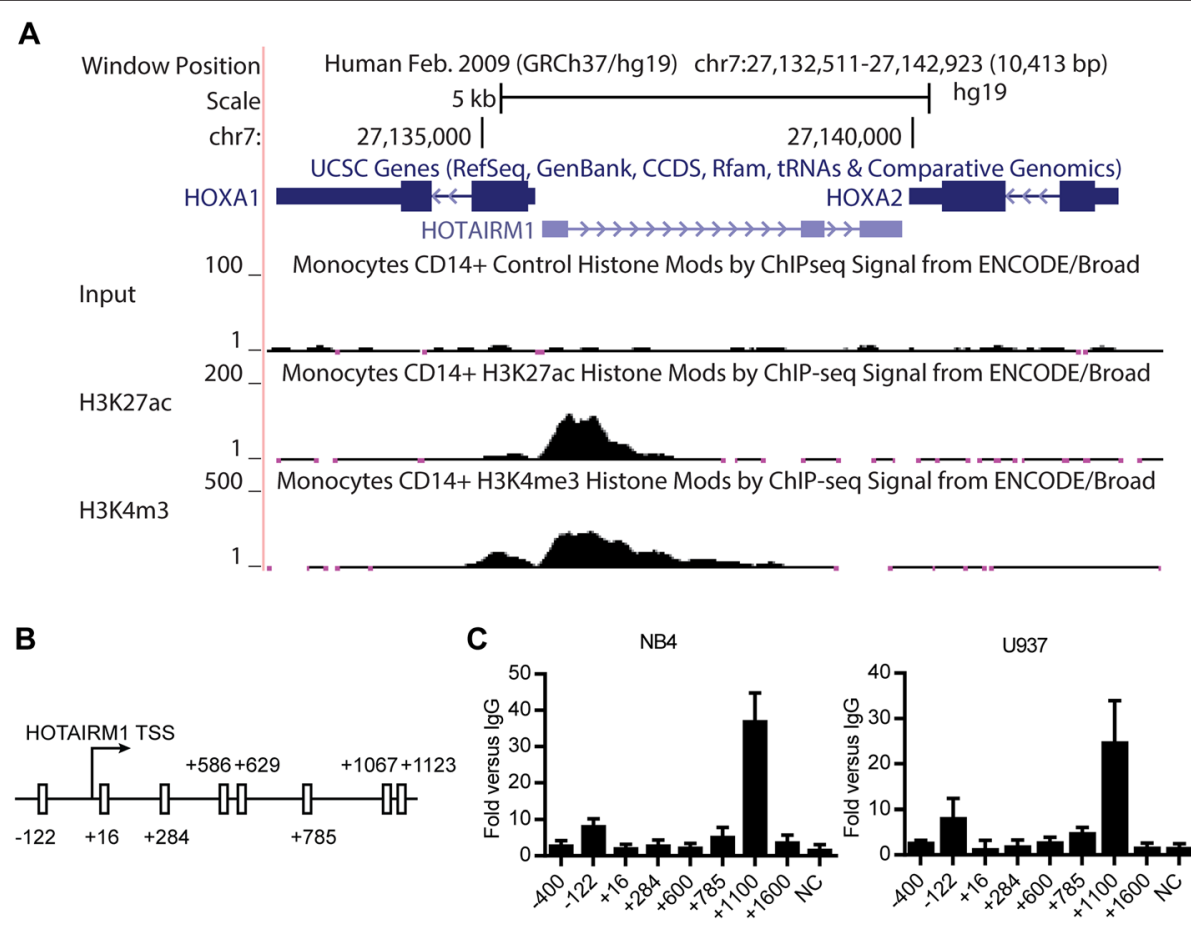

Fig. 2 PU.1 directly binds to the regulatory region of HOTAIRM1. a Genome browser screen shot of the HOTAIRM1 locus. Input (GSM1003475), H3K4me3 (GSM1003536), and H3K27AC (GSM1003559) ChIP-seq signals of CD14+ monocytes were displayed in the UCSC genome browser. b Schematic representation of putative PU.1 binding sites around the promoter region of HOTAIRM1. c PU.1 ChIP-qPCR showing the enrichment of PU.1 in each putative binding site and the negative site in NB4 or U937 cells upon ATRA treatment for $24 \mathrm{~h}$. The data represent the mean of three replicates \pm SD 
A

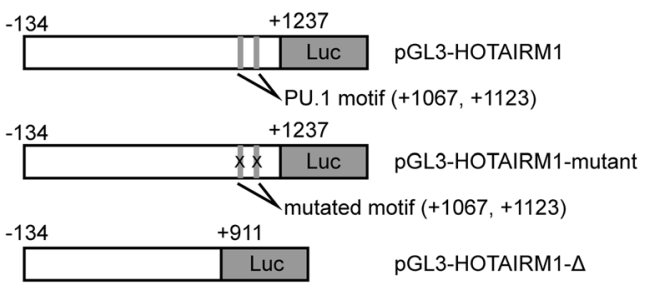

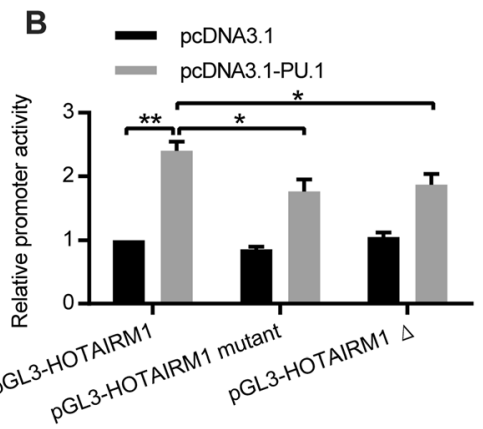

Fig. 3 PU.1 transactivates HOTAIRM1 through the +1100 region in the HOTAIRM1 promoter. a Schematic representation of pGL3-HOTAIRM1 as well as its mutated and truncated forms. b PU.1-transactivated promoter activity of HOTAIRM1. Loss of major PU.1 binding sites significantly impaired PU.1 transactivation on the HOTAIRM1 promoter. The luciferase promoters were co-transfected with equal amount of the pcDNA3.1 or pcDNA3.1-PU.1 vector to HEK-293T cells. The data represent the mean of three replicates \pm SD. ${ }^{*} p<0.05 ;{ }^{* *} p<0.01$

the full-length regulatory region of HOTARIM1. In contrast, the transactivation ability of PU.1 on the regulatory region of HOTAIRM1 was significantly reduced in both PU.1mutated and PU.1-truncated constructs (Fig. 3b). The results indicated that PU.1 could transactivate the HOTAIRM1 promoter, and PU.1 motifs at +1067 and +1123 were essential for the transcriptional activation of HOTAIRM1.

\section{The induction of HOTAIRM1 is dependent on PU.1 during} granulocytic differentiation

Next, we investigated the dependency of HOTAIRM1 on PU.1 in a hematopoietic context. To examine the in vivo regulation by PU.1, we silenced PU.1 in NB4 cells, followed by ATRA treatment for $24 \mathrm{~h}$. To rule out off-target effects, two siRNAs against PU.1 were used and their knockdown efficiencies were around 63 and $74 \%$, respectively (Fig. 4). As a positive control, ITGAM (encoding myeloid integrin CD11b), whose expression is known to rely on PU.1 [17], showed a significant reduction. We used HNRNPH1 as negative control, as its expression level was not affected by the PU.1 level. Both HOTAIRM1 variants were significantly reduced in PU.1 knockdown cells compared with negative control cells (Fig. 4), suggesting that PU.1 was essential for the expression of HOTAIRM1 during granulocytic differentiation.
Additionally, we also detected the effect of arsenic trioxide (ATO), another effective agent used in APL therapy, on HOTAIRM1 expression. We found that ATO had almost no effect on the expression levels of HOTAIRM1 and PU.1 (Additional file 1: Figure S1), further supporting the above observation that the induction of HOTAIRM1 depended on the up-regulation of PU.1.

Low HOTAIRM1 expression is observed in APL cells, which is attributed to the reduced PU.1 expression, rather than the direct binding and repression by PML-RARa

Since HOTAIRM1 expression is up-regulated during ATRA-induced granulocytic differentiation of APL cells, we were interested in HOTAIRM1 expression in APL cells. We first compared the expression of HOTAIRM1 between APL cells and non-APL cells using AML cell lines. In APL patient-derived NB4 cells, the expression abundancy of both HOTAIRM1 and PU.1 was significantly lower than that in non-APL AML cells (U937 and HL60) (Fig. 5a). To validate this finding in a large cohort of patient samples, we utilized previously published transcriptome data [34] to compare their expression between APL patient samples and non-APL AML patient samples. As shown in Fig. 5b, compared to non-APL AML, HOTAIRM1 was noticeably lower in APL patients, which is consistent with a recent clinical study on AML

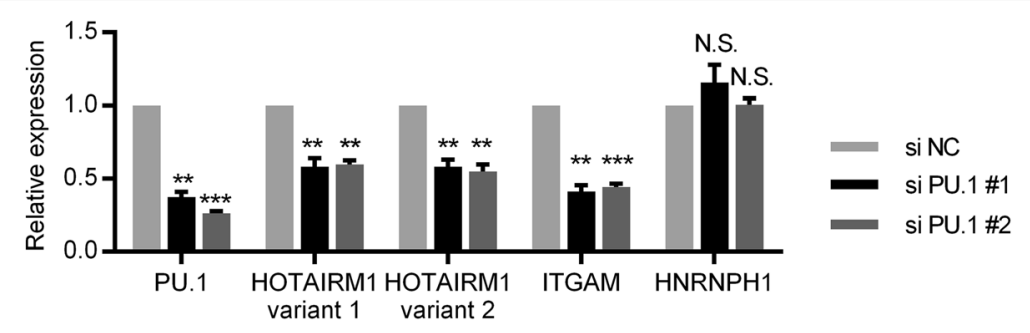

Fig. 4 The induction of HOTAIRM1 is dependent on PU.1 during granulocytic differentiation. The expression of PU.1, HOTAIRM1, ITGAM, and HNRNPH1 after non-targeting or PU.1 siRNA transfection in NB4 cells, with ATRA treatment for $24 \mathrm{~h}$. An average of two control genes (GAPDH and ACTB) was used for normalization. The data represent the mean of three replicates \pm SD. ${ }^{*} p<0.05 ;{ }^{* *} p<0.01 ;{ }^{* *} p<0.001$ 


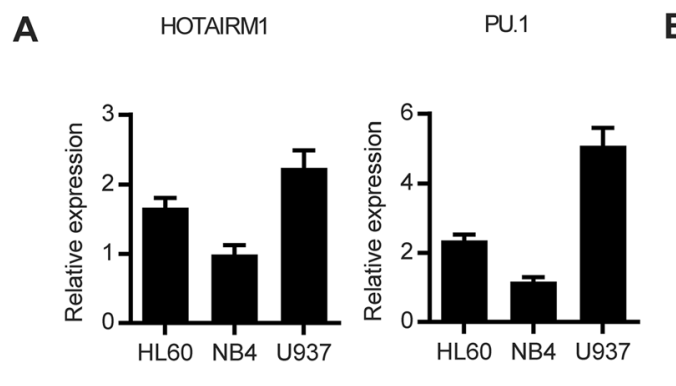

C

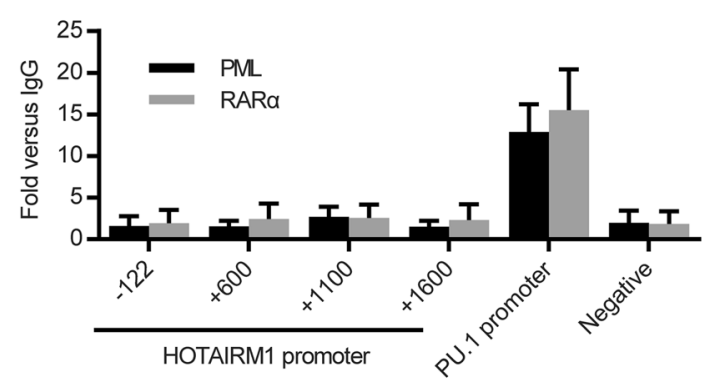

E

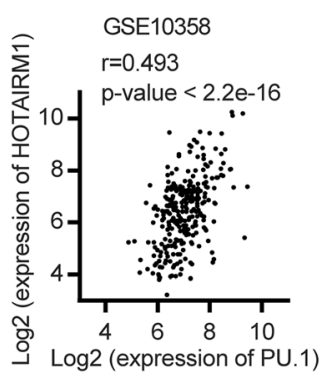

$\mathbf{F}$

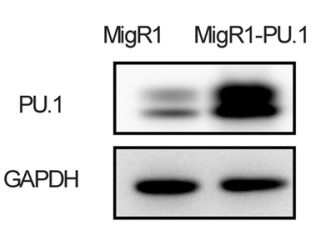

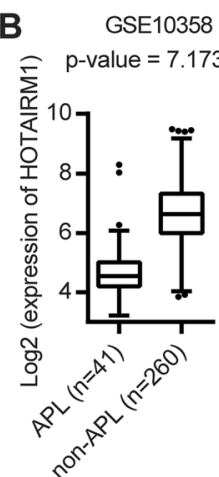

D
GSE10358

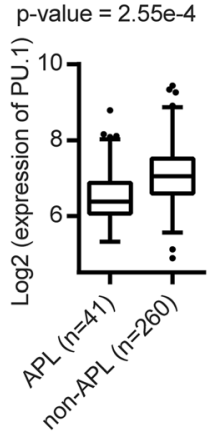

HOTAIRM1 promoter
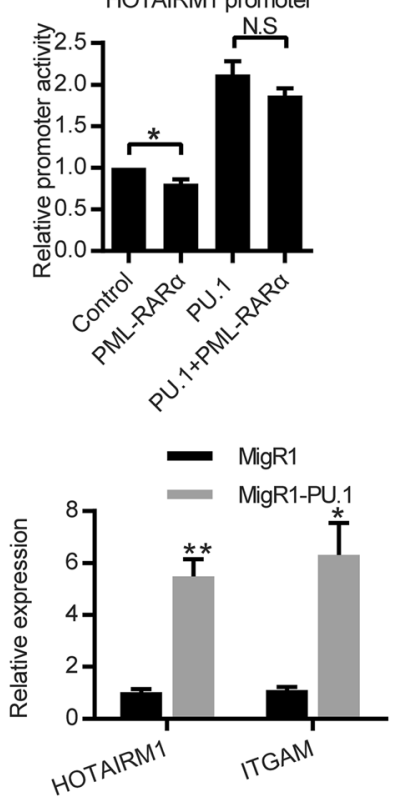

Fig. 5 Low HOTAIRM1 expression is attributed to the reduced PU.1 expression. a HOTAIRM1 and PU.1 mRNA expression levels in representative AML cell lines. b Low levels of HOTAIRM1 and PU.1 in APL patients, as compared with non-APL patients (GSE10358). c ChIP-qPCR showing the binding of PML-RARa on the HOTAIRM1 promoter, the PU.1 promoter (as a positive control), and the negative site. $\mathbf{d}$ The effect of PML-RARa on promoter activity of HOTAIRM1. The pGL3-HOTAIRM1 plasmid was co-transfected with $200 \mathrm{ng}$ expression vectors (pCDNA3.1 alone, PML-RARa alone, PU.1 alone, PU.1 and PML-RARa together, respectively) into HEK-293T cells. e The Pearson correlation between PU.1 and HOTAIRM1 expression in an AML patient cohort (GSE10358). f The up-regulation of HOTAIRM1 after PU.1 overexpression. NB4 cells were harvested at $48 \mathrm{~h}$ post-transduction with the MigR1 empty vector or the MigR1-PU.1 vector. The expression level of PU.1 was detected by western blotting. The mRNA expression levels of HOTAIRM1 and ITGAM were detected by RT-qPCR. GAPDH was used for normalization. The data represent the mean of three replicates \pm SD. ${ }^{*} p<0.05 ;{ }^{* *} p<0.01$

patients [10]. Similarly, PU.1 was also lower in APL, supporting the above cell line-based results.

Low expression of HOTAIRM1 in APL could be due to several possibilities. First, since PML-RAR $\alpha$ generally acts as a repressor on its direct target genes by recruiting co-repressors, such as HDAC1, N-coR, and SMRT [27], HOTAIRM1 expression might be repressed by the direct PML-RAR $\alpha$ binding. To test this possibility, we first examined whether PML-RAR $\alpha$ could directly bind to the regulatory region of HOTAIRM1 using ChIP-qPCR assays. As shown in Fig. 5c, there was no enrichment of either PML or RAR $\alpha$ binding around the regulatory region of HOTAIRM1 in NB4 cells, indicating that PML-RAR $\alpha$ did not directly bind to the HOTAIRM1 promoter to repress its expression. The luciferase reporter assay also showed that PMLRAR $\alpha$ had minimal impact on the promoter activity of HOTAIRM1 (Fig. 5d). Both tests excluded the possibility of the direct binding and repression by PML-RAR $\alpha$.

Second, since we showed that HOTAIRM1 was a PU.1 target gene and was activated by PU.1 (Figs. 2 and 3), and since PML-RAR $\alpha$ is capable of repressing PU.1-regulated genes [23], another possibility might be that PML-RAR $\alpha$ repressed PU.1-dependent transactivation of HOTAIRM1. This possibility was ruled out by the observation that PML-RAR $\alpha$ led to a negligible change 
in PU.1-mediated transactivation through the luciferase reporter assay (Fig. 5d).

Third, low HOTAIRM1 expression could be attributed to the reduced PU.1 expression in APL since PU.1 expression is repressed by PML-RAR $\alpha$ due to disruption of PU.1 autoregulation [23, 35]. To test this possibility, we first examined the correlation between PU.1 and HOTAIRM1 in a large cohort of AML patient samples [34]. We found a significant positive correlation between PU.1 expression and HOTAIRM1 expression $(r=0.493$, $p<2.2 \mathrm{e}^{-16}$ ) (Fig. 5e). To support this finding, we rescued the PU.1 expression by over-expressing PU.1 in PMLRAR $\alpha$-positive NB4 cells. With PU.1 ectopic expression, HOTAIRM1 expression was increased more than fivefold in NB4 cells (Fig. 5f). This also provided an explanation for the up-regulation of HOTAIRM1 in NB4 cells upon ATRA treatment: ATRA restored the expression of PU.1 [29] and then the up-regulated PU.1 transactivated HOTAIRM1.

Taken together, the data suggested that low HOTAIRM1 expression in APL cells was attributed to the reduced PU.1 expression rather than repression by PML-RAR $\alpha$ via the direct binding.

\section{Discussion}

Recently, extensive studies have been carried out to understand the functions of lncRNAs, but fewer studies have focused on the regulation of lncRNAs. In this study, we reveal that the transcription factor PU.1 controls the expression of the IncRNA HOTAIRM1, which acts as a critical noncoding regulator in ATRA-induced granulocytic differentiation of APL cells. On the one hand, we showed that HOTAIRM1 was not a direct ATRA-responsive gene, and the observed up-regulation of HOTAIRM1 induced by ATRA was a secondary event that was dependent on the expression of PU.1 activated by ATRA.

On the other hand, we observed low HOTAIRM1 expression in APL cells and revealed that low expression was attributed to the reduced PU.1 expression, rather than the direct binding and repression of PML-RAR $\alpha$. To support these observations, we provided the evidence that PU.1 constitutively bound to the regulatory region of HOTAIRM1 to transactivate the promoter activity of HOTAIRM1 and identified the two PU.1 motifs responsible for the PU.1-dependent transactivation. These findings enrich our knowledge on the regulation of lncRNAs and the underlying mechanisms of the abnormal expression of lncRNAs involved in APL.

Our first contribution is identifying HOTAIRM1 as a novel target of PU.1. This finding implicates that there still exist unidentified PU.1 targets that are essential for myeloid differentiation. To date, most of the known PU.1 direct target genes participating in myeloid differentiation belong to protein-coding genes [36]. To the best of our knowledge, few have reported the role of PU.1 in the modulation of lncRNAs during myeloid differentiation. Our work presents an example of lncRNAs as the PU.1 target. In addition to HOTAIRM1, there are perhaps hundreds of lncRNA candidates potentially under the transcriptional control of PU.1, among which some are likely correlated with or necessary for myeloid differentiation. Systematic approaches like ChIP-seq and RNAseq would be a powerful tool to globally identify these PU.1 candidate targets. In addition, we also identified the direct binding site of PU.1 at the +1100 downstream of the TSS of the HOTAIRM1 promoter. It is worth mentioning that it was possible that the -122 region might also have a partial impact on the promoter activity of HOTAIRM1, although we focused on the $+1067 /+1123$ locus in the luciferase reporter assay (Fig. 3b). The contribution from the -122 region was probably much less than the $+1067 /+1123$ region, since the in vivo PU.1 binding signals at the $+1067 /+1123$ locus were markedly higher than those at the -122 locus (Fig. 2c).

Second, our work suggests the role of HOTAIRM1 in the PU.1-mediated regulation network during myeloid differentiation. PU.1 regulates differentiation as well as cell cycle and proliferation. For example, exogenous PU.1 expression up-regulates endogenous PU.1 levels in progenitor cells, which is achieved by the induction of cell cycle lengthening, suggesting a positive feedback circuit between PU.1 and the cell cycle in the modulation of myeloid differentiation [37]. PU.1 occupies the CDK6 gene promoter and directly stimulates CDK6 transcription, coordinating proliferation and differentiation [38]. Our work found that HOTAIRM1 was transcriptionally activated by PU.1 and their expression levels were significantly correlated in AML patients. Interestingly, HOTAIRM1 also regulates myeloid differentiation [8] and cell cycle arrest [9]. In addition, HOTAIRM1 modulates CD18 and ITGAM [8], which are known to be transcriptionally controlled by PU.1 $[17,18]$. These findings suggest that HOTAIRM1 may participate in the downstream PU.1-dependent transcription regulation during myeloid differentiation. In another word, HOTAIRM1 may act as a downstream effector of PU.1 by regulating myeloid differentiation-associated genes and cell cycle-related genes.

Third, our research provides insights into the tissue specificity of HOTAIRM1. Tissue specificity is a characteristic of lncRNAs, which usually exhibit a more restricted expression pattern than protein-coding genes [39, 40]. Most recently, IncRNAs have been demonstrated to be dynamically expressed during erythropoiesis and these erythriod-specific transcripts are under the direct transcriptional control of core erythroid transcription factors GATA1, TAL1, or KLF1 [41]. In our work, we 
dissected the regulation control of HOTAIRM1 by PU.1. Since PU.1 is prominent in myeloid cells, increases during myeloid differentiation, and achieves the highest level in mature granulocytes and monocytes [42], our results provide a reason to explain the high tissue specificity of HOTAIRM1 in myeloid cells. To fully explain the myeloid specificity of HOTAIRM1, more studies will be done to learn the whole picture of the combinational and coordinated regulation of HOTAIRM1 by other tissue-restricted transcription factors.

Finally, our work revealed the mechanism underlying the dysregulation of HOTAIRM1 in APL cells. PMLRAR $\alpha$ is a critical factor required for the initiation and development of APL. At the transcriptional level, PMLRAR $\alpha$ represses genes indispensable for normal granulocytic differentiation through multiple ways. First, PML-RAR $\alpha$ interferes with the retinoid acid receptor $\alpha$ (RAR $\alpha)$ signaling. As a potent transcriptional repressor, PML-RAR $\alpha$ directly binds to the retinoic acid response elements (RAREs), recruiting co-repressor proteins such as DAXX, NcoR, and HDAC1 or methylating enzymes such as DNMT1 and DNMT3A, thereby leading to transcriptional repression [43]. Second, PML-RAR $\alpha$ directly binds to PU.1-RAREh (RARE half) binding sites, physically interacts with PU.1, and thus inhibits PU.1-dependent transactivation of myeloid differentiationassociated genes, including SPI1 (PU.1), HCK, PSMB10, and IRF1 [23]. Our study provides an example of the third scenario, which receives less attention in the past compared to the former two scenarios. We did not observe the direct binding of PML-RAR $\alpha$ in the regulatory region of HOTAIRM1. Instead, we found that the dysregulated expression level of HOTAIRM1 was due to the inhibition of PU.1, which suggested the PML-RAR $\alpha-$ suppressive effect on HOTAIRM1 was indirect. Also, this explains the noticeably low level of HOTAIRM1 in APL and suggests that the indirect suppression by PML-RAR $\alpha$ can also be potent. Considering the indispensable role of HOTAIRM1 in myeloid differentiation [8], our work shows that PML-RAR $\alpha$-indirect inhibition of HOTIARM1 contributes to the differentiation block in APL, which provides an additional mechanism for leukemogenesis in APL.

In conclusion, we identify the IncRNA HOTAIRM1 as a novel PU.1-targeted gene and PU.1 mediates the myeloid-specific expression of HOTAIRM1. PU.1dependent transactivation of HOTAIRM1 relies on the two PU.1 motifs at the +1100 region of the HOTAIRM1 promoter. The inhibition of PU.1 by PML-RAR $\alpha$ contributes to the low HOTAIRM1 level in APL cells, which allows us to understand further how the oncofusion protein PML-RAR $\alpha$ blocks myeloid cells at the promyelocytic stage during the development of APL.

\section{Materials and methods}

Cell culture and reagents

HEK-293T cells were cultured in DMEM (Gibco, Carlsbad, CA, USA) containing $10 \%$ fetal bovine serum (Moregate Biotech, Bulimba, QLD, Australia), and NB4, HL60, and U937 cells were cultured in RPMI 1640 (Gibco) supplemented with $10 \%$ fetal bovine serum. Cells were maintained at $37{ }^{\circ} \mathrm{C}$ in a $5 \% \mathrm{CO}_{2}$ incubator. All-trans retinoic acid (Sigma-Aldrich, St Louis, MO, USA) was added to NB4 or U937 cells at a final concentration of $1 \mu \mathrm{M}$.

\section{RNA extraction and qRT-PCR}

Total RNA was extracted using an RNeasy Mini Kit (QIAGEN, Hilden, Germany) according to the manufacturer's directions. Reverse transcription was performed using the SuperScript III Reverse Transcriptase (Invitrogen, Carlsbad, CA, USA). Real-time PCR was performed using the SYBR Green real-time PCR Master Mix (Toyobo, Osaka, Japan) in the Vii7 Real-Time PCR System (Applied Biosystems Inc., Foster City, CA, USA). All primers for quantitative reverse transcription-PCR (qRT-PCR) are listed in Additional file 1: Table S1.

\section{Plasmid construction}

The regulatory region of HOTAIRM1 was amplified using NB4 genomic DNA and then cloned to a pGL3-basic luciferase reporter vector (Promega, Madison, WI, USA). The PU.1-coding sequence was amplified using NB4 cDNA and then cloned to the MigR1 vector (Addgene).

\section{Chromatin immunoprecipitation assay}

Chromatin immunoprecipitation (ChIP) was performed according to the Affymetrix protocol as previously described [44]. The following antibodies were used: anti-PU.1 (\#2258; Cell Signaling Technology, Danvers, MA, USA), anti-RAR $\alpha$ (C-20 X; Santa Cruz Biotechnology, Santa Cruz, CA, USA), and anti-PML (H238 X; Santa Cruz). All primers for ChIP-qPCR are listed in Additional file 1: Table S2.

\section{Luciferase reporter assay}

Luciferase assays were conducted in HEK-293T cells as previously described [25]. Dual-luciferase reporter assays (Promega) were performed $24 \mathrm{~h}$ after transfection by Lipofectamine 2000 (Invitrogen). Renilla luciferase plasmids were used as an internal control for transfection efficiency correction.

\section{siRNA transfection}

The siRNA oligos were transiently transfected to NB4 cells using Nucleofector Kit V (Amaxa Biosystems, Gaithersburg, MD, USA) according to the manufacturer's instructions. PU.1-targeting siRNA oligos matching the sequences: CACUCAAGGCUCUUUGCUU (siPU.1 
\#1) and GAUGUUACAGGCGUGCAAA (siPU.1 \#2), and a negative control siRNA (AGCGUGUAGCUAGCAG AGG), were used. After transfection for $24 \mathrm{~h}$, cells were harvested for qRT-PCR.

\section{Retroviral transduction}

The MigR1 vector (Addgene) was used to transduce NB4 cells for PU.1 overexpression. The preparation of retroviruses and transduction was done as previously described [45]. Briefly, the MigR1-PU.1 vector and the envelope plasmids were co-transfected into HEK-293T cells. After $48 \mathrm{~h}$, retroviral supernatants were collected for transduction. NB4 cells were harvested at $48 \mathrm{~h}$ post-transduction.

\section{Gene expression analysis}

The transcriptome data was provided by Tomasson et al. [34] (GSE10358). Robust multi-array average (RMA) [46] was used to calculate the expression level of HOTAIRM1 and PU.1.

\section{Additional file}

Additional file 1: Figure S1. Arsenic trioxide had minimal effect on the expression levels of HOTAIRM1 and PU.1. Table S1: Primers for RT-qPCR. Table S2: Primers for ChIP-qPCR. (DOCX $35 \mathrm{~kb}$ )

\section{Abbreviations \\ AML: acute myeloid leukemia; APL: acute promyelocytic leukemia; ATRA: all-trans retinoic acid; ChIP: chromatin immunoprecipitation; CHX: cycloheximide; IncRNA: long noncoding RNA; GRT-PCR: quantitative reverse transcription-PCR; TSS: transcription start site.}

\section{Competing interests}

The authors declare that they have no competing interests.

\section{Authors' contributions}

SYW and KKW designed the study. SYW and XLW performed the experiments. MZ and SYW performed the analysis. KKW and SYW wrote the manuscript. KKW, SYW and YZL revised the manuscript. All authors read and approved the final manuscript.

\section{Acknowledgements \\ This work was supported in part by National Natural Science Foundation Grants of China (81530003, 81270625 and 91440114), Shanghai Leading Talent Projects (2015008), the Academic Leader Program of Shanghai Science and Technology Committee (2015137), and the Samuel Waxman Cancer Research Foundation. We also appreciate the help from Xuefei Ma and Jingming Li, who provided experimental input and assistance for the revised manuscript.}

Received: 21 February 2016 Accepted: 25 April 2016

Published online: 04 May 2016

\section{References}

1. Gendrel AV, Heard E. Noncoding RNAs and epigenetic mechanisms during X-chromosome inactivation. Annu Rev Cell Dev Biol. 2014;30:561-80.

2. Goff LA, Rinn JL. Linking RNA biology to IncRNAs. Genome Res. 2015;25: 1456-65.

3. Zhang $H$, Chen Z, Wang $X$, Huang Z, He Z, Chen Y. Long non-coding RNA: a new player in cancer. J Hematol Oncol. 2013;6:37.

4. Huang M, Chen W, Qi F, Xia R, Sun M, Xu T, et al. Long non-coding RNA ANRIL is upregulated in hepatocellular carcinoma and regulates cell apoptosis by epigenetic silencing of KLF2. J Hematol Oncol. 2015;8:50.
5. Wang $P$, Xue Y, Han Y, Lin L, Wu C, Xu S, et al. The STAT3-binding long noncoding RNA Inc-DC controls human dendritic cell differentiation. Science. 2014;344:310-3.

6. Zeng C, Yu X, Lai J, Yang L, Chen S, Li Y. Overexpression of the long non-coding RNA PVT1 is correlated with leukemic cell proliferation in acute promyelocytic leukemia. J Hematol Oncol. 2015;8:126.

7. Dhir A, Dhir S, Proudfoot NJ, Jopling CL. Microprocessor mediates transcriptional termination in genes encoding long noncoding microRNAs. Nat Struct Mol Biol. 2014;2015:1-11.

8. Zhang X, Lian Z, Padden C, Gerstein MB, Rozowsky J, Snyder M, et al. A myelopoiesis-associated regulatory intergenic noncoding RNA transcript within the human HOXA cluster. Blood. 2009;113:2526-34.

9. Zhang X, Weissman SM, Newburger PE. Long intergenic non-coding RNA HOTAIRM1 regulates cell cycle progression during myeloid maturation in NB4 human promyelocytic leukemia cells. RNA Biol. 2014; 11:777-87.

10. Díaz-Beyá M, Brunet S, Nomdedéu J, Pratcorona M, Cordeiro A, Gallardo D, et al. The lincRNA HOTAIRM1, located in the HOXA genomic region, is expressed in acute myeloid leukemia, impacts prognosis in patients in the intermediate-risk cytogenetic category, and is associated with a distinctive microRNA signature. Oncotarget. 2015;6:31613-27.

11. Tenen DG, Hromas R, Licht JD, Zhang DE. Transcription factors, normal myeloid development, and leukemia. Blood. 1997;90:489-519.

12. Voso MT, Burn TC, Wulf G, Lim B, Leone G, Tenen DG. Inhibition of hematopoiesis by competitive binding of transcription factor PU.1. Proc Natl Acad Sci U S A. 1994;91:7932-6.

13. Mossadegh-Keller N, Sarrazin S, Kandalla PK, Espinosa L, Stanley ER, Nutt SL, et al. M-CSF instructs myeloid lineage fate in single haematopoietic stem cells. Nature. 2013;497:239-43.

14. Hohaus S, Petrovick MS, Voso MT, Sun Z, Zhang DE, Tenen DG. PU.1 (Spi-1) and C/EBP alpha regulate expression of the granulocyte-macrophage colony-stimulating factor receptor alpha gene. Mol Cell Biol. 1995;15:5830-45.

15. Zhang DE, Hetherington CJ, Chen HM, Tenen DG. The macrophage transcription factor PU.1 directs tissue-specific expression of the macrophage colony-stimulating factor receptor. Mol Cell Biol. 1994;14:373-81.

16. Smith LT, Hohaus S, Gonzalez DA, Dziennis SE, Tenen DG. PU.1 (Spi-1) and C/EBP alpha regulate the granulocyte colony-stimulating factor receptor promoter in myeloid cells. Blood. 1996:88:1234-47.

17. Pahl HL, Scheibe RJ, Zhang DE, Chen HM, Galson DL, Maki RA, et al. The proto-oncogene PU.1 regulates expression of the myeloid-specific CD11b promoter. J Biol Chem. 1993;268:5014-20.

18. Rosmarin AG, Caprio D, Levy R, Simkevich C. CD18 (beta 2 leukocyte integrin) promoter requires PU.1 transcription factor for myeloid activity. Proc Natl Acad Sci U S A. 1995;92:801-5.

19. Ford AM, Bennett CA, Healy LE, Towatari M, Greaves MF, Enver T. Regulation of the myeloperoxidase enhancer binding proteins Pu1, C-EBP alpha, -beta, and -delta during granulocyte-lineage specification. Proc Natl Acad Sci U S A. 1996;93:10838-43.

20. Vangala RK, Heiss-Neumann MS, Rangatia JS, Singh SM, Schoch C, Tenen DG, et al. The myeloid master regulator transcription factor PU.1 is inactivated by AML1-ETO in t(8;21) myeloid leukemia. Blood. 2003;101:270-7.

21. Huang G, Zhang P, Hirai H, Elf S, Yan X, Chen Z, et al. PU.1 is a major downstream target of AML1 (RUNX1) in adult mouse hematopoiesis. Nat Genet. 2008:40:51-60.

22. Rosenbauer $F$, Wagner $K$, Kutok JL, Iwasaki $H$, Le Beau MM, Okuno $Y$, et al. Acute myeloid leukemia induced by graded reduction of a lineage-specific transcription factor, PU.1. Nat Genet. 2004;36:624-30.

23. Wang $K$, Wang P, Shi J, Zhu X, He M, Jia X, et al. PML/RARa targets promoter regions containing PU.1 consensus and RARE half sites in acute promyelocytic leukemia. Cancer Cell. 2010;17:186-97.

24. Zhu X, Zhang H, Qian M, Zhao X, Yang W, Wang P, et al. The significance of low PU.1 expression in patients with acute promyelocytic leukemia. J Hematol Oncol. 2012;5:22.

25. Zou D, Yang X, Tan Y, Wang P, Zhu X, Yang W, et al. Regulation of the hematopoietic cell kinase (HCK) by PML/RARa and PU.1 in acute promyelocytic leukemia. Leuk Res. 2012;36:219-23.

26. Yang X-W, Wang P, Liu J-Q, Zhang H, Xi W-D, Jia X-H, et al. Coordinated regulation of the immunoproteasome subunits by PML/RARa and PU.1 in acute promyelocytic leukemia. Oncogene. 2014;33:2700-8.

27. de Thé $H$, Chen $Z$. Acute promyelocytic leukaemia: novel insights into the mechanisms of cure. Nat Rev Cancer. 2010;10:775-83. 
28. Wang ZY, Chen Z. Acute promyelocytic leukemia: from highly fatal to highly curable. Blood. 2008;111:2505-15.

29. Mueller BU, Pabst T, Fos J, Petkovic V, Fey MF, Asou N, et al. ATRA resolves the differentiation block in $\mathrm{t}(15 ; 17)$ acute myeloid leukemia by restoring PU. 1 expression. Blood. 2006;107:3330-8.

30. Qian M, Jin W, Zhu X, Jia X, Yang X, Du Y, et al. Structurally differentiated cis-elements that interact with PU.1 are functionally distinguishable in acute promyelocytic leukemia. J Hematol Oncol. 2013;6:25.

31. Consortium EP, Bernstein BE, Birney E, Dunham I, Green ED, Gunter C, et al. An integrated encyclopedia of DNA elements in the human genome. Nature. 2012:489:57-74.

32. Rosenbloom KR, Armstrong J, Barber GP, Casper J, Clawson H, Diekhans M, et al. The UCSC genome browser database: 2015 update. Nucleic Acids Res. 2015;43(Database issue):D670-81.

33. Cartharius K, Frech K, Grote K, Klocke B, Haltmeier M, Klingenhoff A, et al. MatInspector and beyond: promoter analysis based on transcription factor binding sites. Bioinformatics. 2005;21:2933-42.

34. Tomasson MH, Xiang Z, Walgren R, Zhao Y, Kasai Y, Miner T, et al. Somatic mutations and germline sequence variants in the expressed tyrosine kinase genes of patients with de novo acute myeloid leukemia. Blood. 2008;111: 4797-808.

35. Chen H, Ray-Gallet D, Zhang P, Hetherington CJ, Gonzalez DA, Zhang DE, Moreau-Gachelin F, Tenen DG. PU.1 (Spi-1) autoregulates its expression in myeloid cells. Oncogene. 1995;11:1549-60.

36. Friedman AD. Transcriptional control of granulocyte and monocyte development. Oncogene. 2007:26:6816-28.

37. Kueh HY, Champhekar A, Champhekhar A, Nutt SL, Elowitz MB, Rothenberg EV. Positive feedback between PU.1 and the cell cycle controls myeloid differentiation. Science. 2013;341:670-3.

38. Choe KS, Ujhelly O, Wontakal SN, Skoultchi Al. PU.1 directly regulates cdk6 gene expression, linking the cell proliferation and differentiation programs in erythroid cells. J Biol Chem. 2010;285:3044-52.

39. Kutter C, Watt S, Stefflova K, Wilson MD, Goncalves A, Ponting CP, et al. Rapid turnover of long noncoding RNAs and the evolution of gene expression. PLoS Genet. 2012;8:e1002841.

40. Derrien T, Johnson R, Bussotti G, Tanzer A, Djebali S, Tilgner H, et al. The GENCODE v7 catalog of human long noncoding RNAs: analysis of their gene structure, evolution, and expression. Genome Res. 2012;22:1775-89.

41. Alvarez-Dominguez JR, Hu W, Yuan B, Shi J, Park SS, Gromatzky AA, et al. Global discovery of erythroid long noncoding RNAs reveals novel regulators of red cell maturation. Blood. 2014;123:570-81.

42. Chen HM, Zhang P, Voso MT, Hohaus S, Gonzalez DA, Glass CK, et al. Neutrophils and monocytes express high levels of PU.1 (Spi-1) but not Spi-B. Blood. 1995;85:2918-28.

43. Lin RJ, Sternsdorf T, Tini M, Evans RM. Transcriptional regulation in acute promyelocytic leukemia. Oncogene. 2001;20:7204-15.

44. Carroll JS, Liu XS, Brodsky AS, Li W, Meyer CA, Szary AJ, et al. Chromosome-wide mapping of estrogen receptor binding reveals long-range regulation requiring the forkhead protein FoxA1. Cell. 2005;122:33-43.

45. Wang $Y$, Jin W, Jia X, Luo R, Tan Y, Zhu X, et al. Transcriptional repression of CDKN2D by PML/RARa contributes to the altered proliferation and differentiation block of acute promyelocytic leukemia cells. Cell Death Dis. 2014;: : 1431 .

46. Irizarry RA, Hobbs B, Collin F, Beazer-Barclay YD, Antonellis KJ, Scherf U, et al. Exploration, normalization, and summaries of high density oligonucleotide array probe level data. Biostatistics. 2003;4:249-64.

\section{Submit your next manuscript to BioMed Central and we will help you at every step:}

- We accept pre-submission inquiries

- Our selector tool helps you to find the most relevant journal

- We provide round the clock customer support

- Convenient online submission

- Thorough peer review

- Inclusion in PubMed and all major indexing services

- Maximum visibility for your research

Submit your manuscript at www.biomedcentral.com/submit
Biomed Central 